Revue internationale P.M.E.

\title{
Facteurs de risque des stratégies collectives entrepreneuriales
}

Trois études de cas longitudinales (2002-2011)

Risk factors in the entrepreneurial collective strategies

Three longitudinal case studies (2002-2011)

\section{Factores de riesgo de las estrategias empresariales colectivas} Tres casos estudiados longitudinalmente (2002-2011)

\section{Virginie Noireaux}

Volume 28, numéro 1, 2015

URI : https://id.erudit.org/iderudit/1030481ar

DOI : https://doi.org/10.7202/1030481ar

Aller au sommaire du numéro

Éditeur(s)

Editions EMS - In Quarto SARL

ISSN

0776-5436 (imprimé)

1918-9699 (numérique)

Découvrir la revue

Citer cet article

Noireaux, V. (2015). Facteurs de risque des stratégies collectives entrepreneuriales : trois études de cas longitudinales (2002-2011). Revue internationale P.M.E., 28(1), 87-116. https://doi.org/10.7202/1030481ar

\section{Résumé de l'article}

Ce travail s'inscrit dans la continuité des travaux menés sur les stratégies collectives entrepreneuriales (SCE) et s'intéresse à leur dynamique relationnelle. À travers trois cas étudiés de manière longitudinale sur plus de neuf années, nous constatons l'existence d'une dynamique relationnelle qui construit et anime les actions. Les résultats de cette recherche tendent à montrer que la pérennité des SCE semble être étroitement liée à ce processus qui s'articule à trois niveaux : individuel (PME-PMI), groupe et autres parties prenantes. Cette dynamique permet de limiter les risques organisationnels des SCE. Son étude nous permet de dégager un certain nombre d'enseignements pour les acteurs de SCE : une très bonne communication interne, l'identification et la communication continue avec les parties prenantes critiques et institutionnelles, une taille restreinte du comité de pilotage, des décisions orientées vers le plus grand nombre de PME parfois au détriment d'objectifs individuels plus ambitieux. Nous constatons également que l'encastrement institutionnel, très présent les premières années, fait place à un encastrement social, ce qui permet à la SCE d'acquérir une certaine autonomie. 


\title{
Facteurs de risque des stratégies collectives entrepreneuriales : trois études de cas longitudinales (2002-2011)
}

\begin{abstract}
Virginie NOIREAUX
Virginie Noireaux est maître de conférences en sciences de gestion à l'Université d'Auvergne où elle enseigne la stratégie, la logistique et le développement durable. Membre du centre de recherche clermontois en gestion et management (CRCGM), elle travaille sur les stratégies collectives des PME en contexte de développement durable et sur les circuits courts de distribution. Elle est également membre du comité scientifique de l'ADEME (Agence de l'environnement et la maitrise de l'énergie) sur la thématique "déchets et société".

\author{
Clermont Université - Université d'Auvergne \\ École universitaire de management \\ 26, avenue Léon Blum - TSA 20273 \\ 63008 CLERMONT-FERRAND CEDEX 1, France \\ v.noireaux@udamail.fr
}

\begin{abstract}
RÉSUMÉ
Ce travail s'inscrit dans la continuité des travaux menés sur les stratégies collectives entrepreneuriales (SCE) et s'intéresse à leur dynamique relationnelle. À travers trois cas étudiés de manière longitudinale sur plus de neuf années, nous constatons l'existence d'une dynamique relationnelle qui construit et anime les actions. Les résultats de cette recherche tendent à montrer que la pérennité des SCE semble être étroitement liée à ce processus qui s'articule à trois niveaux: individuel (PME-PMI), groupe et autres parties prenantes. Cette dynamique permet de limiter les risques organisationnels des SCE. Son étude nous permet de dégager un certain nombre d'enseignements pour les acteurs de SCE: une très bonne communication interne, l'identification et la communication continue avec les parties prenantes critiques et institutionnelles, une taille restreinte du comité de pilotage, des décisions orientées vers le plus grand nombre de PME parfois au détriment d'objectifs individuels plus ambitieux. Nous constatons également que l'encastrement institutionnel, très présent les premières années, fait place à un encastrement social, ce qui permet à la SCE d'acquérir une certaine autonomie.
\end{abstract}

MoTS CLÉS

Stratégies collectives, PME-PMI, Développement durable

\section{Risk factors in the entrepreneurial collective strategies : three longitudinal case studies (2002-2011)}

\begin{abstract}
This work is interested in the dynamic process of the entrepreneurship collective strategies (ECS). Through three cases of ESC studied during eight years, we notice the existence of a dynamic process which builds and leads the actions. The results of this research tend to show that the perpetuity of the ECS seems to be strictly connected to this process. The dynamic process articulates three levels : individual (SMEs/
\end{abstract}


SMIs), group and others stakeholders. This dynamics allows limiting the organizational risks of the ECS. This study allows us to propose some teachings for the actors of ECS : a very good communication in the group, the identification and the communication with the critical stakeholders, a size restricted by the steering committee, the decisions directed to the large number of SMEs.

\author{
KEYWORDS \\ Collective strategies, SMEs/SMIs, Sustainable development
}

\title{
Factores de riesgo de las estrategias empresariales colectivas : tres casos estudiados longitudinalmente ('2002-2011)
}

\begin{abstract}
RESUMEN
Este trabajo se enmarca, a la vez que continua, los ya realizados sobre Estrategias Empresariales Colectivas (E.E.C.). Así mismo se interesa particularmente en sus Dinámicas Relacionales : a partir de tres casos estudiados longitudinalmente durante algo más de nueve años, se constató la existencia de una dinámica relacional que construye y anima las acciones de E.E.C. Los resultados de esta investigación muestran que las E. E. C. están estrechamente ligadas al proceso relacional y que se articulan a través de tres niveles: individual, grupal y otros grupos de interés. La Dinámica Relacional limita los riesgos organizacionales de las Estrategias Empresariales Colectivas. Por lo tanto, de su estudio se pueden deducir enseñanzas para los Actores de E.E.C. tales como : una buena comunicación interna, identificación de la comunicación continua entre las partes interesadas institucionalmente, tener comités pequeños de toma de decisiones para un grupo numeroso de pequenas y medianas empresas limitando de esta manera objetivos individuales más ambiciosos y se puede constatar igualmente que los ajustes institucionales durante los primeros años, dan lugar a ajustes sociales que repercuten en mayor autonomía para E.E.C.
\end{abstract}

\section{Palabras claves}

Estrategias colectivas, Pequenas y medianas empresas, Desarrollo duradero

\section{INTRODUCTION}

Bien que les PME-PMI soient nombreuses à prendre conscience de l'importance du développement durable (Crocis-CCIP, 2009) et qu'elles soient généralement favorables à l'environnement (Schaper, 2002), une majorité d'entre elles hésite à intégrer la démarche, et les insuccès sont nombreux dans les actions volontaires opérées par les PME (Williamson, Lynch-Wood et Ramsey, 2006). Le manque de ressources humaines et financières (Spence, Ben Boubaker Gherib et Onddoua Biwolé, 2007), le manque d'informations (Clarck, 2000), ainsi que le manque de temps (Hattabou et Louitri, 2011) sont identifiés comme les principaux freins de ces actions. Ces obstacles justifient l'existence de démarches collectives (Berger-Douce, 2010). Ce travail s'inscrit dans la continuité des travaux menés sur les stratégies collectives entrepreneuriales (SCE) (Leyronas et Loup, 2009), et plus particulièrement en contexte environnemental (Berger-Douce, 2010). Une stratégie collective entrepreneuriale peut être définie comme la stratégie d'un ensemble de PME développant des actions communes dans le but de mieux appréhender les menaces ou les opportunités liées à leur développement. Si 
les bienfaits des SCE sont largement décrits dans la littérature (Berger-Douce, 2006 ; Yami, 2006 ; Noireaux et Poirel, 2010), les démarches collectives initiées par les PME se heurtent à de nombreux problèmes de construction et d'animation (Yami, 2003 ; Leyronas et Loup, 2009 ; Berger-Douce, 2006, 2010). Pour expliquer et endiguer ce phénomène, les travaux menés par les chercheurs se rapportent souvent à l'identification des facteurs clés de succès des SCE, mais peu s'intéressent à leur fonctionnement (Yami, 2006). Or, une grande partie du succès de ces organisations s'inscrit dans leur dynamique relationnelle. En effet, un grand nombre de SCE ne perdurent pas dans le temps (Leyronas et Loup, 2009 ; BergerDouce, 2006, 2010), alors qu'elles intègrent au départ l'ensemble des FCS relevés dans la littérature. Dans cette communication, nous cherchons à expliquer la stabilité de ces stratégies au regard de leur dynamique relationnelle. Cette dernière correspond à la capacité d'une SCE existante d'engager des acteurs dans sa démarche afin d'assurer sa survie et son développement. L'objectif est de comprendre la précarité de certaines SCE et de proposer des pistes de réflexion sur le management et l'animation des SCE. L'étude de la littérature nous permettra de présenter et de caractériser notre objet d'étude, les SCE en contexte de développement durable et de comprendre quels sont les principaux risques liés à leur dynamique relationnelle. Puis notre démarche méthodologique sera présentée autour des trois cas de SCE que nous avons étudiés de manière longitudinale. Enfin, nous présenterons notre modèle de dynamique relationnelle et son impact sur les risques organisationnels des SCE.

\section{LES SCE EN CONTEXTE DE DÉVELOPPEMENT DURABLE}

L'analyse des SCE est à l'articulation des approches stratégiques et entrepreneuriales. Nous allons maintenant définir les SCE.

\subsection{Définition et intérêts des stratégies collectives}

Le concept de stratégie collective date des années 1980, mais a connu un regain d'attention depuis quelques années (Roy, 2010). Le fondement conceptuel et l'objectif des stratégies collectives sont présentés dans l'article fondateur d'Astley et Fombrun (1983) qui définissent la stratégie collective comme « la mobilisation commune de ressources et la formulation de l'action au sein de collectivités d'organisation ». Selon cette approche, l'action stratégique permet d'agir sur l'environnement des organisations à travers une "interaction appropriée » (Cyert et March, 1963) afin de se protéger des menaces extérieures ou au contraire, de saisir des opportunités. Ainsi, les PME ont recours aux stratégies collectives, car elles peinent à s'adapter seules à leur environnement (Bollinger et Golden, 1992). La démarche permet de faire face à la turbulence environnementale et de réduire l'incertitude (Bresser et Harl, 1986). Les SCE permettraient ainsi d'augmenter la capacité d'analyse de l'entrepreneur sur son environnement. Or, cette analyse est parfois rendue difficile par manque de temps, de moyens, ou même d'organisation (Vandekerckhove et Dentchev, 2005). Ces stratégies permettent également aux PME d'accéder à des ressources dont elles ne profiteraient pas individuellement. Le propriétaire dirigeant peut opter pour une SCE afin de saisir une ressource extérieure à son activité qu'il ne peut pas acquérir seul (Berger-Douce, 2010). Les SCE per- 
mettent alors de compenser la rareté habituelle des ressources des PME par une mise en commun des ressources ou des actions.

L'émergence de ces stratégies s'expliquerait par le degré élevé d'interdépendance unissant les entreprises. Leur forte interaction sur différents marchés (clients, capitaux, travail...) ou un fort ancrage territorial (Asselineau et Cromarias, 2011) crée une connaissance étroite des entreprises entre elles et favorise le développement de liens coopératifs.

Il est important de souligner que dès le départ, Astley et Fombrun (1983) ont mis l'accent sur les relations entre l'organisation et son environnement. Cette vision oblige à tenir compte d'un ensemble de parties prenantes au sens de Freeman (1984). Les parties prenantes sont définies comme un « individu ou groupe d'individus qui peut affecter ou être affecté par la réalisation des objectifs organisationnels» (Freeman, 1984). Il peut s'agir de fournisseurs, de clients, d'employés, d'investisseurs, des pouvoirs publics, etc. L'étude des parties prenantes a fait l'objet de nombreuses typologies qui reposent sur la nature des parties prenantes (internes ou externes), leurs intérêts, leurs contributions, les relations volontaires ou involontaires qu'elles entretiennent avec l'organisation (Clarkson, 1995).

La satisfaction des parties prenantes apparaît également comme un des facteurs déterminants dans les démarches de développement durable (Bonneveux et Saulquin, 2009). Ces dernières se présentent comme une vraie problématique managériale sous la pression de parties prenantes. Il faut créer de la valeur par la cohésion des acteurs, et c'est le jeu d'acteur qui permet de repérer les actions qui seront prédominantes dans les décisions.

Dans le contexte particulier des stratégies collectives, deux éléments supplémentaires peuvent être identifiés. Le premier concerne la nature même de la stratégie collective qui peut être conçue comme un ensemble de parties prenantes, ces dernières entretiennent des liens plus ou moins directs (Gundolf, Jaouen et Loup, 2006 ; Noireaux et Poirel, 2010). Ces liens déterminent la structure de la pratique collective, et les relations que les membres entretiennent entre eux. Le second concerne les rapports de force entre les différentes parties prenantes d'une SCE. Ceux-ci peuvent être totalement reconfigurés par l'existence de la SCE (Noireaux et Poirel, 2010).

L'étude des parties prenantes est donc centrale dans l'étude des stratégies collectives en contexte de développement durable. Nous allons maintenant caractériser les SCE.

\subsection{Typologie des stratégies collectives}

Astley et Fombrun (1983) proposent quatre types de stratégies collectives, en procédant à une double distinction. La première se réfère au type d'association possible en fonction de la nature des entreprises concernées. Les partenaires sont alors concurrents (soit une association du type « commensal») ou complémentaires (association du type «symbiotique »). La seconde est liée au degré de formalisation de la relation, donnant lieu à une interdépendance « directe » ou « indirecte» (Tableau 1). 
Tableau 1. Typologie des stratégies collectives (Astley et Fombrun, 1983)

\begin{tabular}{lll}
\hline Type d'association & Commensal & Symbiotique \\
\hline Directe & Confédérée & Conjuguée \\
\hline Indirecte & Agglomérée & Organique \\
\hline
\end{tabular}

La problématique qui est la nôtre dans cet article nous positionne dans la colonne des stratégies collectives dites organiques. En effet, les PME qui s'allient sont rarement en situation de concurrence frontale, mais souhaitent développer des actions communes sur la base d'activités complémentaires liées au développement durable, comme la gestion des déchets ou la production d'énergie. Les liens unissant les PME sont plus ou moins formels, car leur implication dans la stratégie n'est pas toujours suivie de la signature d'un contrat.

Loup (2003), s'intéresse plus particulièrement aux stratégies collectives entrepreneuriales et propose une typologie de ces stratégies en fonction de deux critères : l'origine de l'action collective (spontanée, soit " proactive » ou bien institutionnalisée par un acteur extérieur, soit " réactive ") et l'identité des acteurs (trajectoires professionnelles identiques ou ouvertes). Il en résulte quatre types de stratégies : coopérative (proactive/trajectoires professionnelles ouvertes); communautaire (proactive/trajectoires professionnelles identiques); corporatiste (réactive/trajectoires professionnelles identiques) et enfin collaborative (réactive/trajectoires professionnelles ouvertes). Dans notre travail, nous nous intéressons aux stratégies collectives collaboratives, puisque dans les cas étudiés, une institution est à l'origine de la stratégie (cf. partie 2.2.) et que les trajectoires socioprofessionnelles sont ouvertes, puisque les entreprises se regroupent uniquement sur la base de la proximité géographique.

Après avoir défini et caractérisé la SCE dans notre étude, nous allons maintenant présenter les risques liés à leur dynamique relationnelle.

\subsection{Risques liés à la dynamique de la SCE}

La plupart des facteurs clés de succès constatés dans la littérature sont par nature statiques. Ainsi, pour que la SCE émerge, il semblerait qu'il faille remplir un certain nombre de conditions : congruence des objectifs individuels et collectifs, composition cohérente du « groupe projet ", ressources logistiques, interactions avec certaines parties prenantes... (Yami, 2006; Berger-Douce, 2010 ; Bollinger et Golden, 1992).

Si les approches liées aux facteurs clés de succès offrent une photographie à un instant $\mathrm{T}$ des choix des entreprises, elles ne permettent pas d'appréhender comment ces choix évoluent au fil du temps. Or, la pluralité et l'importance des parties prenantes dans les SCE soulignent la difficulté de raisonner en termes de facteurs clés de succès. En effet, les SCE regroupent des acteurs aux profils variés, dont les attentes peuvent être similaires à un instant $\mathrm{T}$, mais contradictoires en $\mathrm{T}+1$. De plus, leur implication peut être continue, mais aussi fluctuante. La littérature sur les relations partenariales, et celle s'intéressant plus particulièrement au SCE, relèvent principalement trois grands types de risques. 
Les deux premiers types de risques émanent du caractère partenarial des relations dans les stratégies collectives : ce sont les risques liés à la performance et les risques relationnels (Das et Teng, 1996).

Le premier risque, que l'on appellera «non-performance ", évoque qu'un partenariat n'atteint pas toujours les objectifs stratégiques que s'étaient fixés les partenaires lors de sa conclusion (Ring et van de Ven, 1992), et ce, même si les efforts de coopération de chacun des alliés sont importants. Ce risque est principalement lié à l'environnement des acteurs (marché...) ou à leurs compétences. Ainsi, l'un des acteurs peut être déçu par les objectifs atteints ou les actions menées et se désengager (Berger-Douce, 2010). Remarquons que ce risque de nonperformance est inhérent à toute stratégie, qu'elle soit réalisée dans un cadre individuel ou dans un cadre collectif.

La seconde menace, inhérente au risque relationnel, est plus spécifique aux stratégies se déroulant dans un cadre collectif. L'instabilité de la stratégie provient de l'incertitude quant au comportement futur du partenaire (Parkhe, 1993). Les alliés peuvent donc faire preuve d'opportunisme (Williamson, 1993), poursuivre leurs propres objectifs et ne pas travailler dans l'intérêt mutuel. De plus, le maintien de plusieurs centres de décision engendre d'incessantes renégociations (Garrette et Dussauge, 1995) susceptibles de freiner, voire de décourager les actions. Les facteurs d'échec des SCE relevés par Berger-Douce (2010) ou par Leyronas et Loup (2009) sont très largement liés à ce risque relationnel : rythmes de progression différents des PME au sein de la stratégie, sentiment de mauvaise gestion des phases de travail dans la durée, modification de l'équilibre entre des objectifs individuels et collectifs.

Le troisième type de risque est présenté dans les travaux de Gundolf, Jaouen et Loup (2006) portant sur les SCE dans le secteur du tourisme. C'est le risque lié à l'encastrement institutionnel qui correspond aux liens existants entre des firmes et des institutions locales (Gundolf, Jaouen et Loup, 2006). Pour eux, cet encastrement est essentiel dans l'étude des SCE et permet d'expliquer, dans une certaine mesure, pour quelles raisons certains collectifs perdurent et augmentent en intensité, alors que d'autres, caractérisés par une ignorance des acteurs, s'étiolent pour finalement cesser. Les institutions, parties prenantes particulières des SCE, permettent de centraliser la gestion du collectif et le coordonnent de manière à ce que les PME puissent s'investir dans leur propre activité. Elles réduisent également le risque de la participation à la stratégie collective perçue par les entrepreneurs, et les encouragent à s'investir dans le projet commun. Ils concluent que si les institutions doivent collaborer avec les acteurs, ces derniers doivent également reconnaître son utilité. Les institutions représenteraient alors un type de parties prenantes spécifiques des SCE par les ressources spécifiques qu'elles peuvent apporter.

Les facteurs d'échec des SCE relevés dans la littérature (Leyronas et Loup, 2009 ; BergerDouce, 2010) sont inhérents à de nombreuses tensions qui apparaissent souvent au cours du temps. C'est pourquoi une vision dynamique et longitudinale de l'organisation est nécessaire. Les conditions initiales déterminent un certain nombre d'éléments qui fixeront les modalités d'exécution des stratégies collectives (partage des rôles, ressources apportées, organisation, résultats attendus...). Néanmoins, au cours du temps, certains risques organisationnels sont susceptibles d'affecter la valeur de la stratégie, et peuvent amener à revoir les objectifs ou l'organisation de la SCE. 


\section{PROTOCOLE MÉTHODOLOGIQUE ET PRÉSENTATION DES CAS ÉTUDIÉS}

\subsection{Une méthodologie de recherche qualitative par études de cas longitudinales}

Notre recherche se fonde sur la méthode de l'étude de cas multi-acteurs (Yin, 1989). Dans l'étude des stratégies collectives, cette méthodologie permet de saisir la complexité des relations, la multiplicité des interactions, des interdépendances et des logiques d'actions (Yami, 2006). Trois études de cas de SCE ont été menées entre 2002 et 2011. Les trois cas concernent initialement la mise en place d'une stratégie collective de gestion de déchets. Les trois SCE présentent les mêmes caractéristiques au départ de l'étude : elles sont chacune portées par une association d'industriels, cette association possède des ressources humaines affectées à la stratégie, le comité de pilotage (Annexe 3 pour la composition des comités de pilotage au cours du temps) est composé de quelques entreprises souhaitant s'investir dans les décisions, et de diverses parties prenantes (ADEME, CCI, prestataires...), l'association qui porte le projet est un acteur déjà important du tissu économique (sauf pour le cas Carros), les objectifs de la stratégie collective sont liés à la réduction des coûts et à une meilleure prise en charge environnementale des déchets. Dans les trois cas, la majorité des entreprises ciblées ne connaissent pas la réglementation liée à la gestion des déchets, ni les techniques de tri. Elles considèrent la gestion des déchets comme coûteuse, mais la majorité d'entre elles connaît mal la valeur de ce coût. Nous avons choisi ici d'étudier des SCE qui avaient à peu près les mêmes caractéristiques au départ et qui répondaient aux FCS identifiés dans la littérature. Leurs caractéristiques sont présentées dans le tableau 2.

TABLEAU 2. PRINCIPALES CARACTÉRISTIQUES DES CAS ÉTUDIÉS

\begin{tabular}{|c|c|c|c|}
\hline & $\begin{array}{l}\text { Profil de l'association } \\
\text { porteuse de la stratégie }\end{array}$ & $\begin{array}{l}\text { Spécificités de la zone } \\
\text { industrielle au départ de } \\
\text { l'étude }\end{array}$ & $\begin{array}{l}\text { Objectifs collectifs au } \\
\text { départ de l'étude }\end{array}$ \\
\hline $\begin{array}{l}\text { Cas } \\
\text { Carros }\end{array}$ & $\begin{array}{l}\text { - Association; } \\
\text { - Peu intégrée : créée } \\
\text { pour la gestion collective } \\
\text { des déchets ; } \\
\text { - Un employé. }\end{array}$ & $\begin{array}{l}\text { - } 400 \text { entreprises et } 7000 \\
\text { emplois ; } \\
\text { - Gisement : entre } 1500 \text { et } 2000 \\
\text { tonnes de D.I.B. }{ }^{1} ; \\
\text { - Fiscalité : TEOM }{ }^{2} \text { et collectes } \\
\text { sans limites de volumes (entre } \\
50 \text { et } 93 \% \text { des entreprises } \\
\text { bénéficient du service de la } \\
\text { collectivité). }\end{array}$ & $\begin{array}{l}\text { - Diminuer le coût ; } \\
\text { - Favoriser le tri ; } \\
\text { - Conformité } \\
\text { réglementaire ; } \\
\text { - Prise en charge } \\
\text { de tous les types de } \\
\text { déchets (hors déchets } \\
\text { dangereux). }\end{array}$ \\
\hline
\end{tabular}




\begin{tabular}{lll}
\hline $\begin{array}{l}\text { Profil de l'association } \\
\text { porteuse de la stratégie }\end{array}$ & $\begin{array}{l}\text { Spécificités de la zone } \\
\text { industrielle au départ de } \\
\text { l'étude }\end{array}$ & $\begin{array}{l}\text { Objectifs collectifs au } \\
\text { départ de l'étude }\end{array}$ \\
\hline
\end{tabular}

\begin{tabular}{|c|c|c|c|}
\hline $\begin{array}{l}\text { Cas Les } \\
\text { Milles }\end{array}$ & $\begin{array}{l}\text { - Association âgée de } \\
\text { six ans ; } \\
\text { - Très intégrée dans le } \\
\text { tissu industriel ; } \\
\text { - Un employé. }\end{array}$ & $\begin{array}{l}\text { - } 1200 \text { PME-PMI dont } 80 \% \text { de } \\
\text { moins de dix salariés et } 21000 \\
\text { emplois ; } \\
\text { - Gisement : } 24300 \text { T de déchets, } \\
\text { dont } 24000 \text { T de D.I.B. et } 3100 \\
\text { T de D.I.S. }{ }^{3} \text {; } \\
\text { - Fiscalité : TEOM sans limites } \\
\text { de volumes (la collectivité } \\
\text { collecte entre } 30 \text { et } 60 \% \text { des } \\
\text { entreprises en fonction des études } \\
\text { qui ont été menées). }\end{array}$ & $\begin{array}{l}\text { - Diminuer le coût ; } \\
\text { - Favoriser le tri ; } \\
\text { - Conformité } \\
\text { réglementaire des } \\
\text { entreprises ; } \\
\text { - Prise en charge } \\
\text { de tous les types de } \\
\text { déchets. }\end{array}$ \\
\hline $\begin{array}{l}\text { Cas La } \\
\text { Garde }\end{array}$ & $\begin{array}{l}\text { - Association âgée de } \\
\text { huit ans ; } \\
\text { - Très intégrée sur la } \\
\text { zone ; } \\
\text { - Trois employés dont } \\
\text { un pour la gestion des } \\
\text { déchets exclusivement. }\end{array}$ & $\begin{array}{l}\text { - } 387 \text { PME-PMI dont } 60 \% \text { de } \\
\text { moins de dix salariés et } 4700 \\
\text { emplois ; } \\
\text { - Gisement : } 8000 \text { tonnes de } \\
\text { D.I.B. ; } \\
\text { - Fiscalité : TEOM sans } \\
\text { limites de volume sur les deux } \\
\text { communes (dont + de } 60 \% \text { par } \\
\text { les collectivités). }\end{array}$ & $\begin{array}{l}\text { - Diminuer le coût ; } \\
\text { - Favoriser le tri ; } \\
\text { - Conformité } \\
\text { réglementaire des } \\
\text { entreprises ; } \\
\text { - Prise en charge } \\
\text { de tous les types de } \\
\text { déchets. }\end{array}$ \\
\hline
\end{tabular}

\footnotetext{
${ }^{1}$ D.I.B. : Déchets industriels banaux.

${ }^{2}$ TEOM : Taxe d'enlèvement des ordures ménagères (montant calculé sur les surfaces bâties).

${ }^{3}$ D.I.S. : Déchets industriels spéciaux.
}

Le choix lié à la thématique de gestion des déchets implique également un certain nombre de caractéristiques communes. La réglementation qui s'applique aux entreprises est la même (Annexe 1), et les parties prenantes sont de même type dans les trois cas. Les parties prenantes représentent classiquement les groupes d'acteurs qui sont susceptibles d'influencer, ou d'être influencés (Freeman, 1984) par la SCE. En matière de gestion des déchets, les principales parties prenantes identifiées par les associations sont présentées dans le tableau 3. 
Tableau 3. Les Parties PRenantes de La gestion des DÉChets des PME-PMI

\begin{tabular}{|c|c|c|}
\hline Partie prenante & Attente & Ressources disponibles \\
\hline $\begin{array}{l}\text { Agence de } \\
\text { l'environnement } \\
\text { et de la maîtrise } \\
\text { de l'énergie } \\
\text { (ADEME) }\end{array}$ & $\begin{array}{l}\text { - Respect de la } \\
\text { réglementation ; } \\
\text { - Susciter, animer, } \\
\text { coordonner des } \\
\text { actions liées à } \\
\text { la protection de } \\
\text { l'environnement. }\end{array}$ & $\begin{array}{l}\text { - Mise à disposition d'informations et d'outils d'ordre } \\
\text { réglementaires et techniques (boîtes à outils et diverses } \\
\text { publications) ; } \\
\text { - Organisation d'échanges d'expériences entre différents } \\
\text { acteurs similaires ou complémentaires (journées } \\
\text { d'informations et d'échanges) ; } \\
\text { - Accompagnement des industriels dans leurs démarches } \\
\text { (participation à des réunions...); } \\
\text { - Aide financière pour les études préliminaires, la } \\
\text { communication et les investissements matériels (sous } \\
\text { réserve d'acceptation des dossiers). }\end{array}$ \\
\hline $\begin{array}{l}\text { Service public } \\
\text { d'enlèvement des } \\
\text { ordures ménagères } \\
\text { (Collectivité) }\end{array}$ & $\begin{array}{l}\text { - Collecte et } \\
\text { traitement des } \\
\text { déchets ; } \\
\text { - Propreté de la } \\
\text { ville et respect } \\
\text { sanitaire. }\end{array}$ & $\begin{array}{l}\text { - Choix de collecter des entreprises (ou non); } \\
\text { - Si elles choisissent de collecter, mise en place de } \\
\text { différentes fiscalités possibles (TEOM, redevance } \\
\text { spéciale) (cf. Annexe } 1 \text { pour la réglementation). }\end{array}$ \\
\hline $\begin{array}{l}\text { Chambres de } \\
\text { commerce (CCI) }\end{array}$ & $\begin{array}{l}\text { - Veiller à la } \\
\text { prise en compte } \\
\text { des intérêts des } \\
\text { entreprises ; } \\
\text { - Faciliter les } \\
\text { rapports entre } \\
\text { entreprises et } \\
\text { administrations. }\end{array}$ & $\begin{array}{l}\text { - Initier des démarches et les animer; } \\
\text { - Accompagner des actions (conseils, liens avec } \\
\text { différents acteurs, organisation de formation...). }\end{array}$ \\
\hline $\begin{array}{l}\text { Direction } \\
\text { régionale de } \\
\text { l'industrie, } \\
\text { la recherche, } \\
\text { l'environnement }\end{array}$ & $\begin{array}{l}\text { - Respect de la } \\
\text { réglementation ; } \\
\text { - Développement } \\
\text { des PME-PMI. }\end{array}$ & $\begin{array}{l}\text { - Aides financières (sous réserve d'acceptation de } \\
\text { dossier); } \\
\text { - Compétences dans l'environnement et dans le } \\
\text { développement des PME-PMI. }\end{array}$ \\
\hline $\begin{array}{l}\text { Entreprises PME- } \\
\text { PMI }\end{array}$ & $\begin{array}{l}\text { - Développer leur } \\
\text { activité ; } \\
\text { - Respect de la } \\
\text { réglementation ; } \\
\text { - Adhérer à des } \\
\text { actions de DD } \\
\text { (pour certaines). }\end{array}$ & $\begin{array}{l}\text { - Choix de gestion des déchets et d'adhésion à la SCE; } \\
\text { - Choix de participer au comité de pilotage et de } \\
\text { s'engager fortement dans les démarches de SCE. }\end{array}$ \\
\hline Prestataires privés & $\begin{array}{l}\text { - Développer leur } \\
\text { activité. }\end{array}$ & $\begin{array}{l}\text { - Mise en place de technologies (pesée embarquée), de } \\
\text { tarifs dégressifs, de tri dans les entreprises... }\end{array}$ \\
\hline
\end{tabular}


La collecte de données s'est faite par entretiens sur une période de huit ans découpée en deux sous-ensembles. D’une part, de 2002 à 2006, les entretiens ont été réalisés une fois par an auprès de plusieurs parties prenantes à la SCE. Cette période correspond à la construction de la stratégie collective. La collecte de données couvrant une période assez longue, certains éléments risquaient d'être réinterprétés par les acteurs en fonction du contexte particulier. C'est pourquoi nous avons choisi d'interroger plusieurs personnes (responsables du projet, prestataires, financeurs, entreprises productrices, consultants). L'interaction des différentes opinions conduit à la dynamique du système et aboutit à un certain équilibre (Thiétart et Forgues, 1995). D’autre part, après 2006, le protocole évolue, car le système est plus stable dans les trois cas étudiés. Les principales décisions qui permettent d'atteindre (ou pas) le projet initial ont été prises, et les organisations sont davantage en phase de routinisation. À partir de cette date, on constate une certaine capitalisation de la première expérience de mise en place d'un service environnemental auprès des PME. Cette capitalisation est alors développée de la même manière sur d'autres projets environnementaux. De plus, les rapports entre les différentes parties prenantes de la gestion des déchets sont stabilisés. Ainsi, le système a abouti à un certain équilibre qui a peu évolué dans les trois cas pendant toute la durée de cette seconde période. C'est pourquoi nous avons réduit la collecte de données à un seul acteur. Un entretien par an a été conduit avec le responsable de l'association porteuse de la stratégie, qui correspond à la personne centrale dans la SCE. Le détail de la planification des entretiens est présenté dans l'annexe 2. Pour compléter ces entretiens, un certain nombre de documents internes et externes ont été étudiés (étude de consultants, étude de la CCI, étude de gisement, consultations, offres des prestataires, coupures de presse...). Ce recueil de données diversifié est aujourd'hui vivement recommandé par les auteurs (Miles et Huberman, 2003) et permet d’assurer la qualité et la fiabilité des données.

En ce qui concerne le traitement des données, une fiche de synthèse a été réalisée par entretien. Sur cette fiche, figure un résumé des thèmes importants qui ont été mentionnés dans l'entretien. Les cinq grands thèmes abordés sont suffisamment larges pour pouvoir être redondants sur toute la durée de l'étude et concernent chacune des périodes : retour sur les principaux éléments de la période précédente, objectifs et sous-objectifs de la SCE, actions menées, organisation des décisions, et perception de l'acteur interrogé sur les objectifs, les attitudes et le choix des autres parties prenantes. Ces fiches permettent d'identifier, par acteur, leurs représentations et leurs évolutions lors des différentes phases. Parallèlement à l'élaboration des fiches, chaque entretien a été enregistré et retranscrit intégralement pour être passé au crible de l'analyse du contenu thématique. Ces retranscriptions, les fiches de synthèse et les documents internes au projet ont fait l'objet d'un codage thématique. Ce codage a débouché sur le découpage de la variable processuelle en étapes clés, conformément aux préconisations d'Ahlström et Karlsson (2009). Ces différentes étapes sont présentées dans le point suivant. 


\subsection{Présentation des cas étudiés}

Le tableau 4 présente les trois cas en fonction du découpage processuel. L'annexe 3 complète la présentation des cas en détaillant les comités de pilotage et leurs évolutions sur toute la durée de collecte des données. La dynamique relationnelle s'articule autour de quatre grandes étapes. La première concerne la genèse du projet de SCE en contexte de développement durable. Elle représente les facteurs déterminants et l'organisation choisie pour gérer le projet. La seconde représente la fixation des objectifs opérationnels et la négociation avec les parties prenantes. Lors de cette phase, les solutions techniques sont choisies. Cette phase correspond à un découpage plus précis des objectifs et répond à une solution spécifique en fonction des attributs et des attitudes des différentes parties prenantes. La troisième correspond à l'évolution des objectifs opérationnels. Dans cette phase, certains objectifs sont renégociés afin de favoriser l'adhésion des entreprises. C'est une phase de réajustement des objectifs. Et enfin, la dernière correspond au développement de la stratégie vers d'autres projets liés au développement durable. Dans cette dernière phase, les acteurs ont capitalisé leur première expérience et développent d'autres actions liées à l'environnement. 
Tableau 4. « Présentation des trois Cas Étudiés », Élaboration Personnelle

\begin{tabular}{|c|c|c|c|}
\hline Phase & Carros & La Garde & Les Milles \\
\hline $\begin{array}{l}\text { Genèse de la } \\
\text { SCE }\end{array}$ & $\begin{array}{l}\text { - Sur proposition de la collectivité, visite } \\
\text { de la zone « Gémenos » qui a mis en } \\
\text { place une gestion collective des déchets ; } \\
\text { - Formation du comité de pilotage ; } \\
\text { - Recrutement d'un chargé de mission; } \\
\text { - Financement d'une étude de gisement } \\
\text { par l'ADEME; } \\
\text { - Présentation du projet à l'ensemble des } \\
\text { entreprises de la zone. }\end{array}$ & $\begin{array}{l}\text { - L'association est très intégrée dans la } \\
\text { zone industrielle. Elle est gestionnaire } \\
\text { de la zone (emplois, locaux, terrains et } \\
\text { gestion du service postal); } \\
\text { - Sur proposition de la CCI, intégration du } \\
\text { tri des déchets dans les compétences de } \\
\text { l'association; } \\
\text { - Financement d'une étude de gisement } \\
\text { par la CCI ; } \\
\text { - Création d'un comité de pilotage. }\end{array}$ & $\begin{array}{l}\text { - La problématique des déchets est } \\
\text { soulevée par un membre du conseil } \\
\text { d'administration de l'association des } \\
\text { entreprises membre de la chambre } \\
\text { régionale de commerce et d'industrie } \\
\text { (CRCI); } \\
\text { - Organisation d'un déjeuner thématique } \\
\text { sur le thème des déchets ; } \\
\text { - Création du comité de pilotage ; } \\
\text { - Financement d'une étude préalable par } \\
\text { l'ADEME. }\end{array}$ \\
\hline Période & 2002 & 1996-1998 & $1998-2002$ \\
\hline $\begin{array}{l}\text { Fixation des } \\
\text { objectifs } \\
\text { opérationnels } \\
\text { et négociations } \\
\text { avec les parties } \\
\text { prenantes }\end{array}$ & $\begin{array}{l}\text { Historique } \\
\text { - Construction du cahier des charges en } \\
\text { fonction de l'étude de gisement ; } \\
\text { - Début des consultations avec les } \\
\text { principaux prestataires de la région; } \\
\text { - Choix du prestataire; } \\
\text { - Actions de communication importantes ; } \\
\text { - Fin } 2004 \text { : début des collectes. }\end{array}$ & $\begin{array}{l}\text { Historique } \\
\text { - Le comité de pilotage fait appel à un } \\
\text { bureau d'études pour proposer différents } \\
\text { scénarios suite aux résultats de l'étude ; } \\
\text { - Une première solution est choisie sur } \\
\text { un scénario modeste qui servira de test et } \\
\text { sera étendu en cas de succès; } \\
\text { - Le projet est retardé. }\end{array}$ & $\begin{array}{l}\text { Historique } \\
\text { - Le comité de pilotage fait appel à un } \\
\text { cabinet de conseil pour proposer différents } \\
\text { scénarios suite aux résultats de l'étude ; } \\
\text { - Une première solution est choisie ; } \\
\text { - Appel d'offres auprès des prestataires ; } \\
\text { - Communication importante, « acte } \\
\text { d'adhésion volontaire des entreprises » } \\
\text { élaboré par le cabinet de conseil ; } \\
\text { - Choix du prestataire ; } \\
\text { - Le projet est retardé. }\end{array}$ \\
\hline
\end{tabular}




\begin{tabular}{|c|c|c|c|}
\hline Phase & Carros & La Garde & Les Milles \\
\hline $\begin{array}{l}\text { Fixation des } \\
\text { objectifs } \\
\text { opérationnels } \\
\text { et négociations } \\
\text { avec les parties } \\
\text { prenantes }\end{array}$ & $\begin{array}{l}\text { Parties prenantes } \\
\text { - La collectivité prend la décision de } \\
\text { mettre en place la redevance spéciale ; } \\
\text { - Démarchage des entreprises par le } \\
\text { prestataire. L'accueil est très mauvais. Le } \\
\text { comité décide que la CCI doit reprendre } \\
\text { cette tâche : informations aux entreprises } \\
\text { sur la démarche et formations au tri ; } \\
\text { - La collectivité quitte le comité de } \\
\text { pilotage en 2004, suite à l'arrêt des } \\
\text { collectes. } \\
\text { Solution technique retenue } \\
\text { Déchets industriels non dangereux, tri de } \\
\text { certains matériaux (papiers cartons, bois, } \\
\text { métaux et films plastiques) et le reliquat } \\
\text { collecté en mélange/pesée embarquée/ } \\
\text { tarification dégressive en fonction du } \\
\text { nombre de points à collecter. }\end{array}$ & $\begin{array}{l}\text { Parties prenantes } \\
\text { - La zone se situe sur deux collectivités } \\
\text { différentes. Une seule souhaite des liens } \\
\text { avec le projet au départ. Elle est donc } \\
\text { invitée ponctuellement au comité de } \\
\text { pilotage, notamment lorsque les sous- } \\
\text { objectifs doivent être discutés. En avril } \\
\text { 1998, la seconde s'intéresse au projet } \\
\text { suite à un changement politique, mais } \\
\text { sans pouvoir y mettre de ressources } \\
\text { (contrairement à la première); } \\
\text { - La collectivité « active » décide de mettre } \\
\text { en place la redevance spéciale en 1999, } \\
\text { mais en 2000, elle abandonne le projet ; } \\
\text { - L'association demande à la collectivité } \\
\text { « active » de se placer comme prestataire } \\
\text { et de mettre en place le tri préconisé par } \\
\text { le bureau d'études, mais les contraintes } \\
\text { sont trop importantes pour la collectivité } \\
\text { et elle doit refuser. } \\
\text { Solution technique retenue } \\
\text { Collecte et tri des papiers cartons et des } \\
\text { plastiques. }\end{array}$ & $\begin{array}{l}\text { Parties prenantes } \\
\text { - La collectivité décide d'exonérer les } \\
\text { entreprises de TEOM et de ne plus } \\
\text { collecter sur la zone ; } \\
\text { - Quelques semaines après le choix } \\
\text { définitif du prestataire, la collectivité } \\
\text { se rétracte sans justification : elle } \\
\text { continuera d'assurer les collectes ; } \\
\text { - Les autres membres du comité de } \\
\text { pilotage décident alors de rompre toute } \\
\text { relation avec la collectivité. } \\
\text { Solution technique retenue } \\
\text { Déchets industriels non dangereux et } \\
\text { dangereux/tri non obligatoire/pesée } \\
\text { embarquée. }\end{array}$ \\
\hline
\end{tabular}




\begin{tabular}{|c|c|c|c|}
\hline Phase & Carros & La Garde & Les Milles \\
\hline Période & 2003-2005 & 1998-2001 & 2003 \\
\hline $\begin{array}{l}\text { Évolution } \\
\text { des objectifs } \\
\text { opérationnels } \\
\text { pour impliquer } \\
\text { les PME-PMI }\end{array}$ & $\begin{array}{l}\text { Objectifs parties prenantes } \\
\text { Un entrepreneur quitte le comité de } \\
\text { pilotage par manque de temps. Le club } \\
\text { communique sur ce départ par courriel } \\
\text { aux entreprises, } 2 \text { semaines après, il est } \\
\text { remplacé ; l'ADEME quitte le comité en } \\
2005 \text { et la CCI en } 2006 \text {. } \\
\text { Objectif technique } \\
\text { Les premiers résultats montrent que le } \\
\text { choix technique s'adapte mal aux TPE, } \\
\text { car les gisements sont trop faibles pour } \\
\text { que le tri soit rentable. Le comité de } \\
\text { pilotage négocie avec le prestataire un } \\
\text { mini forfait avec le tri du papier carton et } \\
\text { le reliquat collecté en mélange. }\end{array}$ & $\begin{array}{l}\text { Objectifs parties prenantes } \\
\text { Après deux ans, le prestataire du comité } \\
\text { de pilotage fait une proposition : la } \\
\text { collecte gratuite du papier carton sur la } \\
\text { zone, car le cours de la matière première } \\
\text { secondaire a évolué et permet de } \\
\text { rentabiliser les frais de transport ; } \\
\text { l'ADEME peut financer les contenants du } \\
\text { tri grâce à une subvention. } \\
\text { Objectif technique } \\
\text { Le tri du papier carton est retenu, car il } \\
\text { permet aux PME PMI de débuter le tri } \\
\text { sans investissement financier, beaucoup } \\
\text { de communication formelle et informelle } \\
\text { sur le projet. }\end{array}$ & $\begin{array}{l}\text { Le comité de pilotage se réunit en } \\
2004 \text { et en } 2005 \text { pour faire le même } \\
\text { constat : le prestataire ne peut faire } \\
\text { concurrence à la collectivité dont } \\
\text { les coûts ne correspondent pas aux } \\
\text { volumes collectés ; en 2006, le } \\
\text { projet est définitivement abandonné ; } \\
2 \text { entrepreneurs quittent le comité. }\end{array}$ \\
\hline
\end{tabular}




\begin{tabular}{|c|c|c|c|}
\hline Phase & Carros & La Garde & Les Milles \\
\hline Période & 2005-2006 & 2001-2004 & 2004-2006 \\
\hline $\begin{array}{l}\text { Vers d'autres } \\
\text { objectifs pour } \\
\text { les PME-PMI }\end{array}$ & $\begin{array}{l}\text { Projet énergie } \\
\text { Le club souhaite mettre en place un projet } \\
\text { lié à l'énergie. Il propose la mise en place } \\
\text { d'un parc éolien. Le projet soulève trop } \\
\text { de réticence. Le club propose alors une } \\
\text { solution avec la technologie photovoltaïque. } \\
\text { Un an après, le club signe un contrat avec } \\
\text { Électricité de France pour l'équipement } \\
\text { des toitures des PME-PMI. L'entreprise } \\
\text { intervient en tiers investisseur : elle décharge } \\
\text { les entreprises de l'investissement, des frais } \\
\text { de maintenance et d'entretien des toitures. } \\
\text { Un an plus tard, } 127 \text { entreprises s'équipent. } \\
\text { Projet déplacement } \\
\text { En 2010, le club s'intéresse à la limitation } \\
\text { des déplacements individuels, lancement } \\
\text { d'une enquête. }\end{array}$ & $\begin{array}{l}\text { Projet DEEE } \\
\text { Organisation d'une journée de } \\
\text { sensibilisation des PME-PMI au tri des } \\
\text { DEEE. Rencontre avec « Micro-orange », } \\
\text { entreprise d'insertion, cette dernière } \\
\text { propose la collecte gratuite des DEEE une } \\
\text { fois par an ; depuis l'action est reconduite } \\
\text { chaque année. } \\
\text { Autre tri } \\
\text { À partir de 2009, la collecte s'étend aux } \\
\text { lampes et néons usagés et aux emballages } \\
\text { souillés. La collecte et le traitement sont } \\
\text { intégralement financés par des aides } \\
\text { locales de la région. }\end{array}$ & $\begin{array}{l}\text { Aucun projet environnemental n'est } \\
\text { proposé par l'association. Les projets } \\
\text { concernent son rôle de gestionnaire de } \\
\text { zone. }\end{array}$ \\
\hline Période & 2006-2011 & 2005-2011 & 2006-2011 \\
\hline $\begin{array}{l}\text { Principaux } \\
\text { résultats chiffrés } \\
\text { des projets }\end{array}$ & $\begin{array}{l}\text { - } 280 \text { adhérents à la gestion collective } \\
\text { (soit plus de } 60 \% \text { des entreprises ciblées) } \\
\text { dont } 37 \% \text { de TPE ; } \\
\text { - } 70 \% \text { des déchets ciblés sont collectés } \\
\text { par la gestion collective et } 400 \text { points de } \\
\text { collecte ; } \\
\text { - Diminution des coûts constatés : de } 10 \\
\text { à } 40 \% \text {; } \\
\text { - } 127 \text { entreprises équipées en } \\
\text { photovoltaïques. }\end{array}$ & $\begin{array}{l}\text { - } 200 \text { entreprises adhérentes au projet } \\
\text { (soit plus de } 50 \% \text { des entreprises de la } \\
\text { zone) ; } \\
\text { - Pas d'impact sur les coûts ; } \\
\text { - Collecte des DEEE entre } 20 \text { et } 45 \mathrm{~m}^{3} \\
\text { par an. }\end{array}$ & Aucun \\
\hline
\end{tabular}




\section{ANALYSE DE LA DYNAMIQUE RELATIONNELLE DE LA SCE}

\subsection{Présentation de la dynamique relationnelle de la SCE}

À travers les trois cas étudiés, nous constatons l'existence d'une dynamique relationnelle qui construit et anime les actions. Cette dynamique s'inscrit dans l'interaction des différentes parties prenantes de la SCE. Cette dynamique relationnelle est représentée par la figure 1. Elle s'articule à trois niveaux : le niveau individuel représente des PME-PMI susceptibles de rentrer dans la SCE, le niveau groupe se réfère au comité de pilotage et le niveau autres parties prenantes. Les interactions entre ces groupes sont par nature mouvantes, ce qui signifie qu'elles peuvent être activées ou pas, et d'une intensité plus ou moins importante. C'est pourquoi nous avons identifié deux sphères à la dynamique relationnelle. La sphère organisationnelle étendue, qui regroupe les liens actifs et inactifs entre les parties prenantes et la sphère organisationnelle restreinte qui représente uniquement les liens actifs entre les membres de la SCE. Les liens actifs représentent un engagement dans la stratégie, cet engagement pouvant prendre différentes formes (participation financière, aide technique, contribution aux collectes...). Ces deux sphères sont par nature perméables, c'est-àdire que le lien entre une partie prenante et le groupe peut être activé à tout moment. Dans le cas «La Garde », une des collectivités décide de rejoindre la SCE en 1998 suite à un changement de politique interne qui fait du développement durable une politique prioritaire. C'est également le cas de "Micro-Orange » qui devient une partie prenante critique en 2005 en collectant les déchets électriques et électroniques. Avant cette date, ces déchets spécifiques ne rentraient pas dans les actions visées par la SCE.

Le niveau individuel représente les PME-PMI qui participent ou sont invitées à intégrer la SCE. À ce niveau, les objectifs poursuivis par les SCE et la volonté d'engagement influencent largement la stratégie. Ce sont les objectifs fixés par le groupe, ainsi que la communication, qui sont susceptibles ou non d'engager de nouveaux acteurs dans la démarche. En effet, il est possible que certaines PME choisissent de ne pas s'engager dans la SCE, car les objectifs ne correspondent pas à leurs attentes à un instant T. Dans le cas de Carros, les gisements des TPE ne correspondaient pas au cadre négocié par la SCE. En 2006, le comité de pilotage négocie un contrat particulier pour les très petits gisements. Après une forte campagne de communication, les TPE adhèrent. En 2011, $37 \%$ des adhérents correspondent à de très petits gisements. La communication a eu un rôle important, d'autant plus que certaines des TPE s'étaient déjà renseignées avant 2006 sur la SCE. La communication permet de garder le lien avec les PME ciblées, et d'engendrer de nouvelles adhésions à la SCE soit en faisant évoluer les actions proposées, soit par l'évolution des attentes des PME.

Le groupe (comité de pilotage de la SCE) représente la communauté "socio-économique » (Gundolf, Jaouen et Loup, 2006), ou le groupe projet (Berger-Douce, 2010). Il regroupe certains acteurs particulièrement engagés qui analysent les différentes actions possibles, fixent les objectifs et mettent en œuvre les décisions. L'influence du groupe est centrale, et l'articulation entre les objectifs fixés et les actions menées déterminent le succès (ou l'échec) des SCE (Gundolf, Jaouen et Loup, 2006 ; Berger-Douce, 2010). L'interaction des membres du groupe permet d'éviter les dérives liées à l'atteinte d'objectifs personnels au sein du comité. 
Les entreprises qui s'impliquent dans les comités de pilotage sont généralement très sensibles aux objectifs de la SCE (d'où leur engagement important). La tentation de manœuvrer la SCE pour atteindre leurs objectifs personnels peut être un risque significatif d'échec de la stratégie collective. Dans le cas " La Garde », les objectifs initiaux ont été complètement renégociés. La gestion de l'ensemble des déchets non dangereux, qui était initialement l'objectif principal, est partiellement suspendue pour faire place à la prise en charge de déchets plus spéciaux comme les DEEE ou les néons. Dans un contexte difficile d'implication des parties prenantes institutionnelles, le comité a décidé de réaliser des actions moins ambitieuses, mais qui répondent aux besoins réels des PME. Inversement, le cas « Les Milles », qui rencontre la même difficulté, n’a pas su identifier de nouveaux objectifs, ni de nouvelles actions, malgré les réunions du comité de pilotage de 2004 à 2006. Dans ce cas, l'interaction entre les membres a toujours été plus difficile : il a fallu trois ans au comité pour conclure qu'il ne pourrait pas finaliser le cahier des charges, par manque de compétences.

\section{Figure 1. Dynamique Relationnelle des SCE}

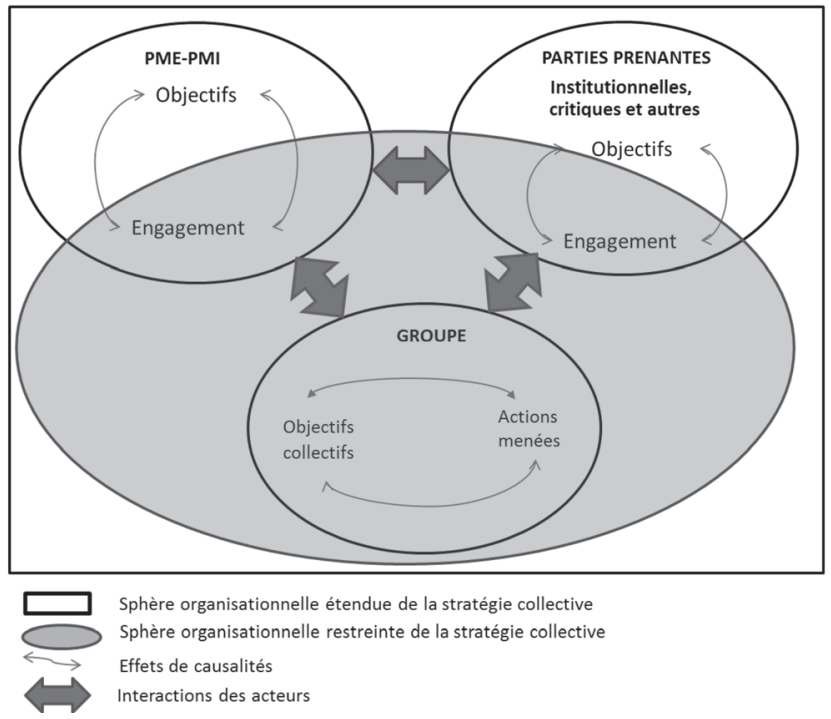

Les autres parties prenantes correspondent aux autres organisations, individus ou groupes d'acteurs liés de manière plus ou moins formelle à la SCE. Elles peuvent être différentes en fonction des actions menées et apportent un soutien en matière de ressources, d'expertise ou d'accès à un réseau (Noireaux, 2006). Leurs objectifs sont par nature partiellement liés aux actions menées par la SCE, même s'ils ne sont pas toujours concordants. Trois types de parties prenantes peuvent être identifiés. Le premier concerne les parties prenantes « institutionnelles » : ADEME, État, Région, CCI... Elles ont pour vocation d'aider les démarches collectives et/ou les PME. Le second concerne les parties prenantes critiques, ces dernières décident librement de coopérer (ou non) à la SCE. Elles ont une influence majeure sur la stratégie, car elles contrôlent des ressources essentielles et effectuent des activités qui peuvent interagir avec les actions menées par la SCE. Dans la gestion des déchets, ce sont typiquement les prestataires privés et les collectivités. En matière de gestion des déchets, la collectivité, 
partie prenante institutionnelle, est également une partie prenante critique puisque c'est elle qui gère historiquement les déchets des entreprises et qu'elle a la possibilité de garder cette gestion en adaptant son service et sa fiscalité. Dans la gestion de l'énergie, ce sont les entreprises qui commercialisent l'électricité (principalement Électricité de France en France). Le troisième représente les acteurs qui sont susceptibles d'interférer avec la démarche ou de devenir une partie prenante critique ou un membre du groupe. À titre d'exemple, l'entreprise Électricité de France, dans le cas de Carros, est devenue une partie prenante critique et un membre du groupe, lorsque le comité de pilotage a décidé de créer un projet sur l'énergie. Le tableau 5 compare la dynamique relationnelle des trois cas étudiés et l'annexe 4 présente les variables utilisées pour caractériser les relations. 
TAbleau 5. «COMPARAison DeS Dynamiques Relationnelles »

\begin{tabular}{|c|c|c|c|c|c|c|c|c|}
\hline \multirow[t]{2}{*}{ Cas } & \multicolumn{2}{|l|}{ PME-PMI } & \multicolumn{2}{|l|}{$\mathbf{P P}$} & \multicolumn{2}{|l|}{ Groupe } & \multirow{2}{*}{$\begin{array}{l}\text { Interaction } \\
\text { PME-Groupe }\end{array}$} & \multirow{2}{*}{$\begin{array}{l}\text { Interaction PP- } \\
\text { Groupe }\end{array}$} \\
\hline & Objectifs & Engagement & Objectifs & Engagement & Interactions & Actions & & \\
\hline Carros & $\begin{array}{l}\text { - Services } \\
\text { adaptés } \\
\text { aux } \\
\text { attentes } \\
\text { - DD } \\
\text { - Coût }\end{array}$ & Important & $\begin{array}{l}\text { - Réglementation } \\
\text { - Coût } \\
\text { - DD }\end{array}$ & $\begin{array}{l}\text { Important } \\
\text { car répondant } \\
\text { aux politiques } \\
\text { internes }\end{array}$ & $\begin{array}{l}\text { - Régulière } \\
\text { - } 1 \text { conflit } \\
\text { majeur entre } \\
\text { les membres }\end{array}$ & $\begin{array}{l}\text { - Déchets } \\
\text { - Énergie } \\
\text { - Déplacement }\end{array}$ & $\begin{array}{l}\text { Communication } \\
\text { intensive, } \\
\text { fréquente et } \\
\text { de plus en plus } \\
\text { informelle }\end{array}$ & $\begin{array}{l}\text { Communication } \\
\text { fréquente jusqu'en } \\
2006\end{array}$ \\
\hline $\begin{array}{l}\text { La } \\
\text { Garde }\end{array}$ & $\begin{array}{l}\text { - Services } \\
\text { adaptés } \\
\text { aux } \\
\text { attentes } \\
\text { - DD } \\
\text { - Coût }\end{array}$ & Important & $\begin{array}{l}\text { - Attractivité } \\
\text { de la zone } \\
\text { industrielle } \\
\text { - DD } \\
\text { - Coût }\end{array}$ & $\begin{array}{l}\text { - Inconstant } \\
\text { d'une PP } \\
\text { critique } \\
\text { - Rupture } \\
\text { d'une autre PP } \\
\text { critique }\end{array}$ & $\begin{array}{l}\text { - Régulière } \\
\text { - Pas de } \\
\text { conflits entre } \\
\text { les membres }\end{array}$ & $\begin{array}{l}\text { - Gestion de la } \\
\text { zone } \\
\text { - Déchets }\end{array}$ & $\begin{array}{l}\text { Communication } \\
\text { intensive, } \\
\text { fréquente et } \\
\text { informelle dès le } \\
\text { départ }\end{array}$ & $\begin{array}{l}\text { Communication } \\
\text { peu fréquente }\end{array}$ \\
\hline $\begin{array}{l}\text { Les } \\
\text { Milles }\end{array}$ & $\begin{array}{l}\text { - Services } \\
\text { adaptés } \\
\text { aux } \\
\text { attentes } \\
\text { - Coût } \\
\text { (-DD) }\end{array}$ & $\begin{array}{l}\text { Aucun, } \\
\text { service non } \\
\text { mis en place }\end{array}$ & $\begin{array}{l}\text { - Attractivité } \\
\text { de la zone } \\
\text { industrielle } \\
\text { - DD } \\
\text { - Coût }\end{array}$ & $\begin{array}{l}\text { Rupture d'une } \\
\text { PP critique }\end{array}$ & $\begin{array}{l}\text { - Irrégulière } \\
\text { - } 5 \text { conflits } \\
\text { majeurs }\end{array}$ & $\begin{array}{l}\text { - Gestion de la } \\
\text { zone } \\
\text { - Échec du } \\
\text { projet de } \\
\text { gestion des } \\
\text { déchets }\end{array}$ & $\begin{array}{l}\text { Communication } \\
\text { ponctuelle }\end{array}$ & $\begin{array}{l}\text { - Communication } \\
\text { peu fréquente } \\
\text { - Rupture totale } \\
\text { de communication } \\
\text { d'une PP critique }\end{array}$ \\
\hline
\end{tabular}


Nous constatons que les cas où la dynamique relationnelle est restée active au cours du temps (Carros et Les Milles), la stratégie a abouti à des actions concrètes de collectes pour les PME. Inversement, dans le cas «Les Milles », la dynamique relationnelle est moins active et les actions sont abandonnées. Dans la partie suivante, nous verrons comment la dynamique relationnelle peut agir sur les risques organisationnels liés aux SCE.

\subsection{Dynamique relationnelle et risques organisationnels}

\section{DYNAMIQUE RELATIONNELLE ET NON-PERFORMANCE}

La non-performance de la SCE peut se traduire par l'abandon du projet ou par un faible taux d'adhésion des PME ciblées. Les raisons de cette absence de performance peuvent être multiples.

Une première raison se trouve dans la non-atteinte de l'objectif économique de la SCE. Or, dans l'ensemble des cas, la gestion économique des déchets était un objectif majeur des SCE. En matière d'environnement, nous constatons que les objectifs de la grande majorité des PME-PMI sont avant tout liés à la maîtrise des coûts, ce qui converge avec les autres études de SCE en contexte de développement durable (Berger-Douce, 2010), et qui s'explique également par le fait que la SCE ne concerne pas ici le cœur de métier des PME impliquées dans la stratégie. La plupart des entreprises interrogées identifient la gestion des déchets comme une charge supplémentaire obligatoire, et non comme un problème environnemental lié à leur activité. Dans le cas "Les Milles », la solution de la SCE est plus onéreuse que celle proposée par la collectivité. Le projet est donc abandonné. Cependant, à coût équivalent, une majorité d'entreprises est prête à mettre en place des démarches de tri. Dans le cas « La Garde », les actions sont centrées sur le volontariat des entreprises puisque les solutions mises en place sont gratuites pour les entreprises. Cette démarche connaît un franc succès, puisqu'environ $50 \%$ des entreprises ont adhéré à la collecte sélective du papier carton, et que la journée de collecte de déchets spécifiques est reconduite chaque année. Dans les deux cas « Les Milles » et " La Garde ", la collectivité collecte les déchets des entreprises avec une fiscalité avantageuse pour elles (calcul de la TEOM non lié aux volumes produits). Mais contrairement au cas «Les Milles », la zone de "La Garde » a su adapter sa stratégie à son environnement, et plus particulièrement à ses difficultés relationnelles avec la collectivité. Dans un contexte difficile d'implication des parties prenantes critiques, le comité a décidé de réaliser des actions moins ambitieuses (tri des déchets pouvant être collectés gratuitement : papier/carton, DEEE, néons et lampes usagés), mais qui répondent aux besoins réels des PME. Inversement, le cas "Les Milles ", qui rencontre la même difficulté, n’a pas su identifier de nouveaux objectifs, ni de nouvelles actions. L'interaction entre les membres a toujours été plus difficile, ce qui peut s'expliquer par le fait que les membres du comité sont beaucoup plus nombreux que dans les autres cas ( 16 membres au lieu de 7 pour La Garde et 8 pour Carros). La multiplication des membres entraîne un certain «immobilisme » du comité, car les propositions se multiplient, les décisions sont plus longues à négocier, et les réunions plus difficiles à planifier. 
Dans le cas « Carros », la mise en place d'un mini-forfait adapté financièrement aux TPE suscite l'adhésion d'une centaine d'entreprises l'année suivante. Le coût financier reste l'un des principaux freins à la mise en place des démarches de développement durable dans les PME (Spence, Rutherfoord et Blackburn, 1998). Les SCE doivent donc trouver un équilibre entre les objectifs poursuivis et les coûts supplémentaires qu'impliquent les actions menées. De plus, les objectifs poursuivis doivent prendre en compte l'environnement extérieur, c'est-àdire les stratégies individuelles des parties prenantes critiques, dont les actions sont susceptibles d'interférer sur les actions de la SCE. Il semblerait que cette adaptation est plus facile à mettre en place dans un groupe relativement restreint et où sont représentées les parties prenantes critiques.

L'intégration des parties prenantes critiques au comité de pilotage semble fondamentale pour la viabilité de la SCE, et ce, même si leurs objectifs divergent avec ceux de la SCE. Si elles ne souhaitent pas intégrer le comité de pilotage, il semble important d'inscrire la communication avec elles dans la durée, car un changement de politique interne, ou d'environnement, peut impacter le déroulement des actions de la SCE (positivement ou négativement). Ainsi, dans le cas « La Garde », les réunions du comité de pilotage entre 2004 et 2006 permettent de garder les liens entre les acteurs. Parallèlement, l'environnement économique du prestataire évolue (augmentation du cours du papier carton sur le second marché). Les contacts annuels entre les membres ont permis au prestataire de faire une proposition originale, basée sur le coût. Si la communication avait été rompue, le prestataire n'aurait pas pu proposer cette solution.

\section{DYNAMIQUE RELATIONNELLE ET RISQUE RELATIONNEL}

Le risque relationnel représente une détérioration des relations d'une manière brutale ou progressive, soit entre les différents types de parties prenantes, soit à l'intérieur du groupe.

La détérioration des relations est liée à un changement de stratégie individuelle d'une partie prenante. Si le changement concerne une des PME ciblées, elle se retirera de la démarche, ce qui n'aura pas de conséquence importante sur la SCE, à partir du moment où les actions menées par cette dernière correspondent à un besoin collectif et que les actions de communication auprès des PME sont suffisantes. Dans le cas de "Carros", trois PME s'étaient engagées au départ à adhérer aux actions de la SCE, puis se sont rétractées. Pour l'une d'entre elles, les besoins du dirigeant étaient différents de la solution proposée par la SCE. L'entrepreneur souhaitait trier des déchets alimentaires issus de sa production. Les actions proposées par la SCE ne correspondent donc pas à ses besoins. Pour deux autres, leur prestataire de services s'est «aligné » sur la solution proposée par la SCE. À prix et services identiques, les entrepreneurs préféraient garder leur prestataire historique.

Le changement brutal d'objectif peut également concerner une partie prenante critique (cas de la collectivité dans le cas « La Garde » et «Les Milles »). Dans ce cas, ce sont les objectifs de la SCE qui peuvent être impactés par leurs décisions. Le groupe doit alors redéfinir ses actions afin de s'adapter à cette turbulence. Si l'adaptation ne se fait pas (comme dans le cas des « Milles »), les actions de la SCE sont mises en échec. Nous n’avons pas observé dans les cas étudiés une évolution des objectifs des acteurs institutionnels. Ce cas doit être plus rare, car les objectifs de ces acteurs sont généralement actés sur des périodes relativement longues (4 à 5 ans). Cependant, les conséquences pourraient être importantes pour la SCE, non pas 
en termes d'objectifs, mais en termes de financement et de fonctionnement. En effet, ce type de parties prenantes s'engage généralement à aider les SCE en apportant des ressources pour faciliter la mise en place des actions ou le fonctionnement du groupe.

À l'intérieur du groupe, il est également possible de voir apparaître au cours du temps une dégradation des relations. Dans le cas « Carros », lors de l'élaboration du projet sur "l'énergie verte », la solution technique retenue (les panneaux photovoltaïques) est mal acceptée par deux membres du comité de pilotage. L'installation de panneaux photovoltaïques est considérée comme moins performante que la solution initiale proposée (parc d'éolien). Cependant, le projet éolien soulevait trop de réticence de la part des entreprises : la place, la propriété des terrains et la crainte des nuisances sonores. La technologie photovoltaïque est mieux perçue par les PME. Ici, le groupe considère l'acceptabilité des actions comme centrale. Les interactions entre les membres favorisent un nombre d'adhérents potentiellement élevé, plutôt que l'atteinte d'objectifs individuels des entreprises qui s'impliquent dans le comité de pilotage. Suite à cette décision, un des deux entrepreneurs " est aujourd'hui en retrait sur les décisions, mais souhaite tout de même rester... Il est passif dans les décisions, mais n'est pas non plus source de freins ou d'inerties; si cela avait été le cas, je pense qu'on lui aurait demandé de partir» (porteur du projet). Vraisemblablement, l'entrepreneur attend une nouvelle action du groupe pour s'investir davantage ou décider de quitter la SCE.

\section{DYNAMIQUE RELATIONNELLE ET ENCASTREMENT INSTITUTIONNEL}

L'encastrement institutionnel correspond aux liens existants entre des firmes et des institutions locales (Gundolf, Jaouen et Loup, 2006). Dans les trois cas étudiés, l'encastrement institutionnel était présent au départ de l'étude. Ce sont des institutions qui sont à l'origine de la réflexion sur les déchets. Si l'encastrement institutionnel est important au départ de la SCE, nous constatons qu'il s'élargit au cours du temps pour faire place à un encastrement social.

Dans le cas "Carros », les liens entre le comité de pilotage et les institutions sont très forts jusqu'en 2006 ; à partir de cette date, le projet sur les déchets rentre dans une phase de " routinisation ", et les institutions ne sont plus aussi indispensables. La collectivité s'est désengagée progressivement de la gestion des déchets des entreprises : à l'initiative du projet, elle attend l'engagement du prestataire sur les coûts/services pour officialiser son désengagement. À partir de 2003, elle annonce son retrait des collectes sur la zone et l'exonération de TEOM pour les entreprises qui participeront aux collectes de la gestion collective. Les autres entreprises ne seront pas exonérées. Cette exonération « choisie " permet à la collectivité de s'assurer de la conformité réglementaire des collectes et d'éviter les problèmes de déchets sur la voie publique dont elle a la responsabilité. En 2004, l'exonération est progressivement mise en place pour les nouveaux adhérents. En 2005, la CCI a terminé les études de gisements et les formations au tri (cette action est reprise par l'employé du club des entreprises pour les PME souhaitant tardivement intégrer la démarche), l'ADEME a terminé ses financements (études et contenants). Le rôle des institutions est donc moins prégnant qu'en phase de mise en place de la SCE. Les institutions ne sont plus représentées en tant que membre permanent dans le comité de pilotage à partir de 2006 (voir Annexe 3). À partir de 2006, le projet sur l'énergie est proposé par le groupe. Ce projet ne fait pas appel à des ressources institutionnelles, même si l'ADEME a eu un rôle de conseil ponctuel en tant qu'expert au sein du comité de pilotage. On constate donc que l'encastrement institutionnel est moins fort au cours du temps. Il 
semble élargi à un encastrement social. L’encastrement social signifie que les interactions économiques s'entremêlent avec des interactions sociales (lien familial, amical, associatif...) et que, in fine, la qualité de ces dernières influence la transaction (Granovetter, 1985). Ici, la dynamique de la SCE crée des liens sociaux forts entre les différentes parties prenantes qui lui permettent de se développer. L'encastrement institutionnel, bien que présent devient mineur, au profit des liens qui sont tissés localement entre les PME et le groupe. Dans le cas de « La Garde », nous constatons le même phénomène. Dans ce cas, l'encastrement social était déjà fort au départ de notre étude. En effet, l'association de la zone développait de nombreuses actions : gestion des offres et demandes d'emplois, gestion des locaux et des terrains disponibles, et aussi gestion du service postal pour l'ensemble des PME. L'association connaissait tous les entrepreneurs, et les liens les unissant étaient déjà importants. Dans la mise en place de leurs actions, le rôle des institutions a été moins prégnant que dans les deux autres cas. Elles ont été consultées pour définir le projet et les aides pouvant être accordées, notamment sur le projet de tri des déchets non dangereux, car la politique des collectivités locales était essentielle. Lors de la mise en place du projet de collecte des DEEE, les institutions n'ont pas été consultées. Pour celui qui est relatif aux lampes et néons usagés, les institutions ont été consultées pour des financements qui permettent la collecte " gratuite " pour les PME. Ici, la dynamique de la SCE implique l'utilitarisme du groupe (Jackson et Rogers, 2007 ; Powell, White, Koput et Owen-Smith, 2005). Les liens entre les différents types d'organisation se créent et se défont en fonction de l'intérêt économique des organisations.

Ainsi, si l'encastrement institutionnel semble essentiel au départ d'une SCE, il est élargi au fil du temps par un encastrement social entre le groupe et les PME. Cet encastrement social permet à la SCE d'atteindre un certain niveau d'indépendance par rapport aux institutions, ces dernières pouvant générer une certaine dépendance de sentier, limitant les choix possibles des acteurs en matière d'actions (Belussi, Sammarra et Rita-Sedita, 2008). L'élargissement de cet encastrement permettrait donc de développer plus facilement d'autres objectifs et actions que ceux qui sont proposés par les politiques institutionnelles.

\section{CONCLUSION}

Les résultats de cette recherche tendent à montrer que la pérennité des stratégies collectives entrepreneuriales semble être étroitement liée à la dynamique relationnelle qui anime les actions. Cette dynamique s'articule à trois niveaux : individuel, groupe et parties prenantes. Elle permet de faire face aux risques organisationnels des SCE : non-performance, risque relationnel et absence d'encastrement. Pour Bresser et Harl (1986), le risque sous-jacent à une stratégie collective pour l'entreprise est de perdre sa propre dynamique concurrentielle et d'avoir des difficultés à s'adapter à son environnement. Ainsi, les stratégies collectives porteraient en elles les conditions de leur propre remise en cause et seraient inévitablement limitées dans le temps. Nous arguons dans ce travail que la dynamique relationnelle de l'ensemble des parties prenantes impliquées permet de pallier ce problème. En effet, la dynamique relationnelle permet d'engager différents acteurs dans différentes actions, ce qui limite les problèmes individuels d'adaptation. Chaque PME peut s'engager dans une action, ou dans plusieurs, en fonction de ses besoins et de ses spécificités. Il s'avère également que l'encastrement institutionnel, donné comme un facteur de risque des stratégies collectives 
(Gundolf, Jaouen et Loup, 2006), se restreint au fil du temps sans compromettre la viabilité de la SCE. Essentielle au départ, notamment parce que beaucoup d'acteurs institutionnels ont une bonne connaissance des PME pouvant s'engager dans la démarche, leur expertise devient moins essentielle lorsque les liens groupe-PME deviennent plus importants. À cette phase, les PME échangent directement avec le groupe projet, ce qui permet de faire émerger d'autres besoins. Les acteurs institutionnels sont toujours présents, mais ont davantage un rôle de facilitateur, à travers les éventuels financements. L'encastrement institutionnel est élargi à un encastrement social entre les différentes parties prenantes. Cet encastrement social est la conséquence de la dynamique relationnelle. Cette dernière crée des liens entre les parties prenantes et assure la dynamique du système.

Sur le plan managérial, ce travail révèle l'importance d'identifier les différentes parties prenantes liées à une stratégie collective. Lorsque les acteurs s'engagent dans une telle stratégie, les différents types de parties prenantes doivent être identifiés. Au cours de la mise en place de la stratégie, les liens avec les parties prenantes critiques sont essentiels, même si ces dernières poursuivent des objectifs individuels qui vont à l'encontre de l'objectif initial de la SCE. La compréhension des objectifs poursuivis par les parties prenantes critiques permet à la SCE d'adapter ses objectifs en fonction de son environnement. De plus, si les liens avec les parties prenantes critiques sont essentiels, la communication avec les autres parties prenantes et avec les PME est également importante tout le long du déroulement de la stratégie. Cette communication permet de bénéficier de ressources (PP institutionnelles notamment), et de proposer des objectifs en adéquation avec les attentes des PME qui peuvent potentiellement entrer dans la SCE. La dynamique relationnelle de l'ensemble des membres d'une SCE permet alors de pérenniser la stratégie en engageant différents membres sur différents types d'objectifs. Enfin, il s'avère que la constitution du groupe et la communication entre les membres sont essentielles. Ce groupe doit être de taille restreinte, et composé de membres souhaitant développer des actions qui profitent aux plus grands nombres de PME, quitte à renoncer à leurs convictions personnelles. Les autres membres du groupe doivent veiller à cet aspect, même si cela conduit à écarter un membre.

Cette étude, comme toutes celles qui sont basées sur des études de cas, est marquée par une validité externe relativement restreinte. D’une part, la stratégie collective développée ici ne concerne pas le cœur de métier des entreprises. Si la problématique liée à la coopétition et à ses conséquences est très présente dans les analyses portant sur les stratégiques collectives (Dagnino et Rocco, 2009), il convient de noter que dans le cas que nous étudions, elle n'est pas prégnante dans la mesure où la coopération des PME impliquées porte sur des activités de soutien. Cette caractéristique ouvre deux voies de recherches principales. Premièrement, l'étude de la dynamique relationnelle dans le cadre de stratégie collective portant sur le cœur de métier des entreprises. D’autre part, le développement des stratégies collectives que nous qualifierons " de soutien », qui a pour objectif de proposer un certain nombre de services aux PME pour augmenter leur attractivité individuelle (emploi, certification...) et/ou l'attractivité géographique d'une zone. 


\section{ANNEXE 1. RÉGLEMENTATION LIÉE À LA GESTION DES DÉCHETS INDUSTRIELS}

En France, la loi-cadre du 15 juillet 1975, convertie dans l'article L 541-2 du Code de l'environnement, impute la gestion et l'élimination des déchets au producteur dans la lignée du principe du pollueur payeur. Elle fixe également les dispositions légales de cette gestion. Ces lois s'appliquent à tous les types de déchets et à l'ensemble des activités logistiques qui s'y rapportent.

La réglementation pesant sur les producteurs peut être résumée de la manière suivante :

- L’obligation d'assurer ou de faire assurer l'élimination de ses déchets dans des conditions qui évitent les effets néfastes sur l'environnement (article L 541-2 du Code de l'environnement) ;

- L'obligation de justifier la destination finale des déchets, ce qui implique la traçabilité (articles L 541-2 et L 541-7 du Code de l'environnement);

- La mise en place d'obligations spécifiques en matière de collecte, de stockage et de transport des déchets d'emballage (décret $n^{\circ} 94-609$ du 13 juillet 1994), ainsi que pour d'autres déchets (les déchets dangereux : décret nº 2005-635 du 30 mai 2005);

- L'interdiction de mise en décharge de déchets autres que les déchets « ultimes » (article L 541-24 du Code de l'environnement) qui ne peuvent être traités dans les conditions économiques et techniques du moment. Cette interdiction demande un système complexe exigeant transports, tris et valorisations multiples.

Les D.I.B. possèdent un statut particulier. En effet, ils peuvent, si la collectivité le désire, être collectés dans le cadre du service public d'enlèvement des ordures ménagères (Code de l'environnement, article L 541-21). La loi fixe également les deux modalités de financement du service, qui doit être facturé aux industriels proportionnellement au service rendu. Soit la collectivité collecte avec le TEOM, taxe calculée à partir des propriétés bâties, soit elle met en place une redevance spéciale, calculée sur les volumes collectés.

Annexe 2. Collectes de donnéES

\begin{tabular}{|c|c|c|c|c|c|c|c|c|c|c|c|c|}
\hline \multirow{3}{*}{$\begin{array}{l}\text { Source } \\
\text { Responsable du projet }\end{array}$} & \multicolumn{6}{|c|}{$2002-2006$} & \multicolumn{6}{|c|}{ 2006-2011 } \\
\hline & \multicolumn{2}{|c|}{ Carros } & \multicolumn{2}{|c|}{$\begin{array}{l}\text { Les } \\
\text { Milles }\end{array}$} & \multicolumn{2}{|c|}{ La Garde } & \multicolumn{2}{|c|}{ Carros } & \multicolumn{2}{|c|}{$\begin{array}{l}\text { Les } \\
\text { Milles }\end{array}$} & \multicolumn{2}{|c|}{ La Garde } \\
\hline & 1 & 4 & 1 & 4 & 1 & 3 & 1 & 4 & 1 & 2 & 1 & 3 \\
\hline Prestataire & 2 & 4 & 1 & 2 & 1 & 1 & 0 & 0 & 0 & 0 & 0 & 0 \\
\hline Financeur & 2 & 3 & 2 & 4 & 1 & 2 & 0 & 0 & 0 & 0 & 0 & 0 \\
\hline Entreprise productrice & 1 & 4 & 1 & 2 & 1 & 3 & 0 & 0 & 0 & 0 & 0 & 0 \\
\hline $\begin{array}{l}\text { Autre (salarié, } \\
\text { consultant...) }\end{array}$ & 1 & 2 & 0 & 0 & 1 & 2 & 0 & 0 & 0 & 0 & 0 & 0 \\
\hline
\end{tabular}




\begin{tabular}{|c|c|c|c|c|c|c|}
\hline \multirow[b]{3}{*}{ Source } & \multirow{2}{*}{\multicolumn{3}{|c|}{$2002-2006$}} & & & \\
\hline & & & & \multicolumn{3}{|c|}{ 2006-2011 } \\
\hline & Carros & $\begin{array}{l}\text { Les } \\
\text { Milles }\end{array}$ & La Garde & Carros & $\begin{array}{l}\text { Les } \\
\text { Milles }\end{array}$ & La Garde \\
\hline $\begin{array}{l}\text { Étude de consultants } \\
\text { (commandée lors de } \\
\text { la mise en place de } \\
\text { l'action collective) }\end{array}$ & oui & oui & oui & non & non & non \\
\hline $\begin{array}{l}\text { Documents internes au } \\
\text { projet (études, compte } \\
\text { rendu, contrats...) }\end{array}$ & oui & oui & oui & oui & non & oui \\
\hline $\begin{array}{l}\text { Source secondaire } \\
\text { publique }\end{array}$ & oui & oui & oui & oui & oui & oui \\
\hline
\end{tabular}

Durée des entretiens : de 45 min à 3 h 10. Colonne de gauche, nombre de personnes interrogées $(0 ; 1 ; 2)$; Colonne de droite, nombre d'entretiens. 


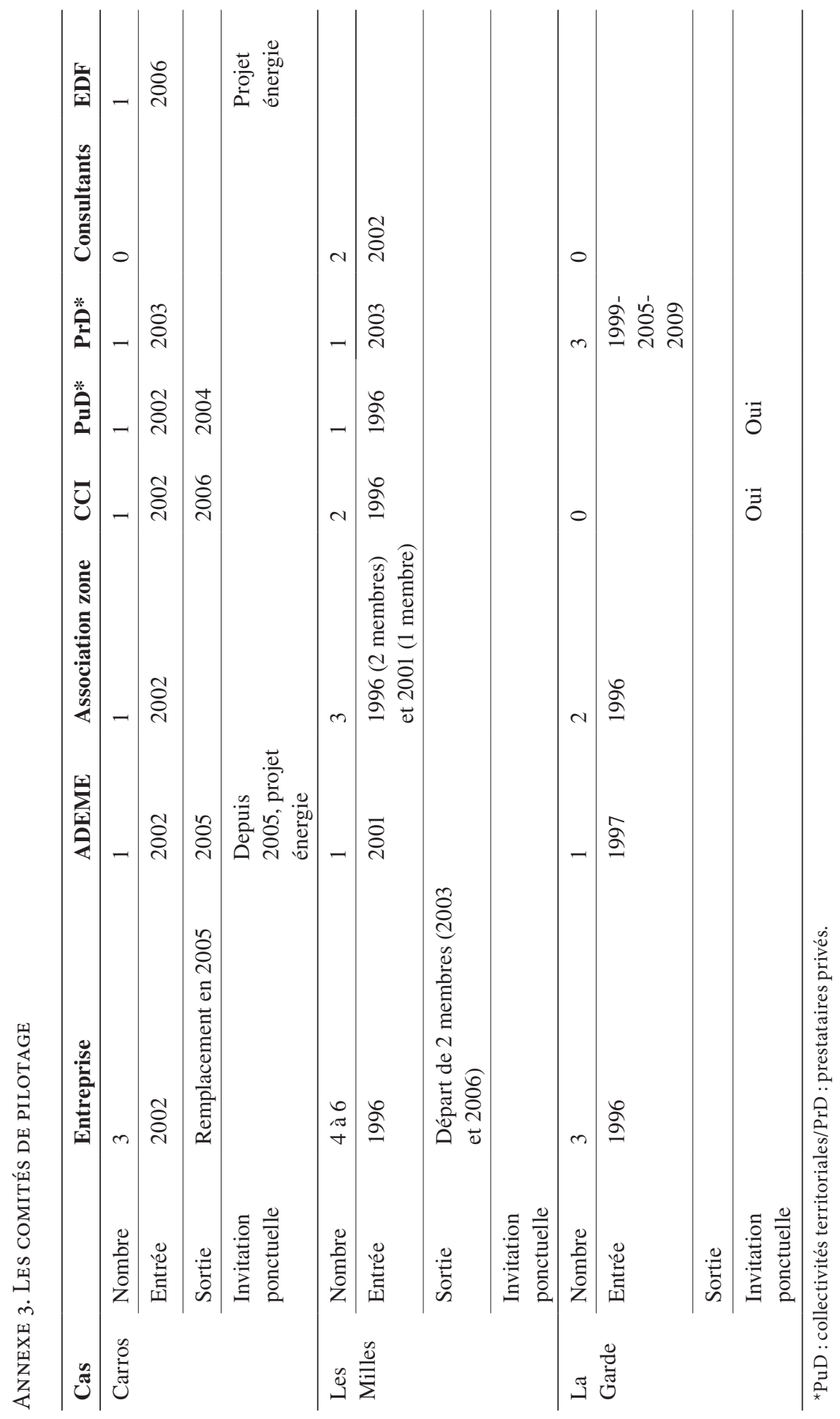


ANNEXe 4. VARIABLES UtiliséES POUR CARACTÉRISER LES RELATIONS ENTRE LES CAS

\begin{tabular}{ll}
\hline Qualificatif utilisé dans le tableau 5 & Variable(s) et seuil(s) \\
\hline Objectifs & 3 principaux objectifs les plus cités par type d'acteurs \\
\hline Engagement & - Important $:<30 \%$ des PME ciblées adhèrent aux \\
PME-PMI & projets proposés \\
PP & Important $:$ mise à disposition de moyens humains \\
& et/ou financiers \\
& - Inconstant $:+$ de 2 changements de politique sur la \\
& période étudiée \\
& - Rupture $: 1$ changement de politique \\
\hline Interaction groupe & Régulière $: \leq 1$ réunion par trimestre \\
& - Irrégulière $: \geq 1$ réunion par trimestre \\
& - Conflits majeurs $:$ conflits qui entravent la prise de \\
& décision sur une durée de plus d'un trimestre \\
\hline Interaction intergroupe & Communication intensive $:<3$ supports de \\
& communication (journaux internes, affichage, \\
& presse locale, radio...) \\
& - Communication informelle $:$ Contacts directs et non \\
& programmés \\
& - Communication ponctuelle $: \leq 2$ supports sur une \\
& période $\leq 1$ mois \\
& - Communication fréquente $:<1$ fois par trimestre \\
\hline
\end{tabular}

\section{RÉFÉRENCES}

Ahlstrôm, P. et Karlsson, C. (2009). Longitudinal field studies. Dans C. Karlsson (dir.), Researching Operations Management (p. 196-236). New York, Routledge.

Asselineau, A. et Cromarias, A. (2011). Les stratégies collectives sont-elles toujours applicables dans un "milieu » ? Une réflexion à partir du contre-exemple de la coutellerie thiernoise. Revue management \& avenir, 10(50), 137-152.

Astley, W.G. et Fombrun, C.J. (1983). Collective strategy : social ecology of organizational environments. Academy of Management Review, 8(4), 576-587.

Belussi, F., Sammarra, A. et Rita-Sedita, S. (2008, juin). Industrial district evolutionary trajectories: localized learning diversity and external growth. Conférence de Druid. Copenhage, Danemark.

BERGER-DouCE, S. (2006). La démarche collective, un outil d'appropriation du management environnemental par les PME ? Revue des sciences de gestion, 1(54), 19-36.

BERgER-Douce, S. (2010). La fabrique d'une stratégie collective entrepreneuriale orientée environnement : l'expérience d'un club de dirigeants des TIC. Revue internationale PME, 23(3-4), 123-154.

Bollinger, M.J. et Golden, P.A. (1992). Interorganizational and collective strategies in small firms : environmental effects and performance. Journal of Management, 18(4), 695-715. 
Bonneveux, E. et SAUlquin, J.Y. (2009). L’appropriation de la RSE par les dirigeants de PME. Le réseau comme vecteur de l'apprentissage managérial. Revue management \& avenir, 3(23), 170-186.BRESSER, R.K. et Harl, J.E. (1986). Collective strategy : vice or virtue? Academy of Management Review, 11(2), 408-427.

Clarck, G. (2000). Developing better systems for communications, environmental best practices in small business. Dans R. Hillary (dir.), Small and Medium-Sized Enterprises and the Environment (p. 128-147). Sheffields, Business Imperatives, Greenleaf Publishing.

Clarkson, M.B. (1995). A stakeholder framework for analyzing and evaluating corporate social performance. Academy of Management Review, 20(1), 92-117.

CROCIs-CCIP (2009). Le développement durable dans les PME-PMI de la région parisienne - baromètre 2009. Récupéré le 5 octobre 2011 du site : http://www.crocis.ccip.fr.

Cyert, R.M. et March, J.G. (1963). A behavioral theory of the firm. Englewood Cliffs, New Jersey, Prentice-Hall.

Dagnino, G.B. et Rocco, E. (2009). Coopetition strategy : theory, experiments and cases. Londres/ New York, Routledge.

DAs, T.K. et Teng, B.S. (1996). Risk types and inter-firm alliance structures. Journal of Management Studies, 33(6), 827-843.

Freeman, R.E. (1984). Strategic management : a stakeholder approach. Cambridge, Pitman.

Garrette, B. et Dussauge, P. (1995). Les stratégies d'alliance. Paris, Les Éditions d'Organisation.

Granovetter, M. (1985). Economic action and social structure : the problem of embeddedness. American Journal of Sociology, 91(3), 481-510.

Gundolf, K., Jaouen, A. et Loup, S. (2006). La place des institutions locales dans les stratégies collectives : le cas des activités touristiques. Revue française de gestion, 8(176), 141-157.

Hatтaвou, A. et Louitri, A. (2011). Développement durable et management des PME : une analyse en termes de proximité : illustration par un cas du secteur textile-habillement. Management \& avenir, 3(43), 122-142.

Jackson, M. et Rogers, B. (2007). Meeting strangers and friends of friends : how random are social networks? American Economic Review, 97(3), 890-915.

Leyronas, C. et Loup, S. (2009). Processus de performance des stratégies collectives en TPE : proposition d'un cadre d'analyse. Dans K. Messeghem et M. Polge (dir.), Entrepreneur et dynamiques territoriales (p. 267-277). Cormelles-le-Royal, France, Éditions EMS.

Loup, S. (2003, 3-6 juin). Un essai de clarification des stratégies entrepreneuriales collectives : application au secteur des métiers d'art. $11^{\mathrm{e}}$ Conférence de l'Association internationale de management stratégique. Les Côtes de Cartage, Tunisie.

Miles, M.B. et Huberman, A.M. (2003). Qualitative data analysis : a sourcebook of new methods. Londres, Sage Publications.

Noireaux, V. (2006). L'émergence du leadership reconnu dans le canal de distribution inversée : le cas des déchets industriels (thèse de doctorat en sciences de gestion). Université de la Méditerranée, AixMarseille II.

Noireaux, V. et Poirel, C. (2010). La logistique des déchets industriels entre rivalités et partenariats : une analyse des formes collectives de contre-pouvoir. Logistique et management, 18(2), 35-49. 
PARKhe, A. (1993). Strategic alliances structuring : a game theory and transaction cost examination of interfirm cooperation. Academy of Management Journal, 36(4), 794-829.

Powell, W., White, D., Koput, K. et Owen-Smith, J. (2005). Network dynamics and field evolution: the growth of interorganizational collaboration in the life sciences. American Journal of Sociology, 110(4), 1132-1205.

RING, P.S. et VAN DE Ven, A.H. (1992). Structuring cooperative relationship between organization. Strategic Management Journal, 13(5), 483-498.

Roy, P. (2010). Les nouvelles stratégies concurrentielles (Repères). Paris, La Découverte, 128 p.

SCHAPER, M. (2002). The challenge of environmental responsibility and sustainable development : implications for SME and entrepreneurship academics. Dans Urs Füglistaller (dir.), Radical changes in the world : will SME soar or crash (p. 541-553). Saint Gallen, KMU HSG.

SPENCE, L.J., RUtherfoord, R. et Blackburn, R. (1998). Small firms and environmental issues in the UK and the Netherlands : a literature review and research agenda. Dans SBRC Monograph (p. 5-98). Royaume-Uni, Kingston University.

Spence, M., Ben Boubaker Gherib, J. et Onddoua Biwolé, V. (2007). Développement durable et PME : une étude exploratoire des déterminants de leur engagement. Revue internationale PME, 20(34), $17-42$.

Thiétart, R.A. et Forgues, B. (1995). Chaos theory and organization. Organization Science, 6(1), 19-31.

Vandekerckhove, W. et Dentchev, N.A. (2005). Network perspective on stakeholder management: facilitating entrepreneurs in the discovery of opportunities. Journal of Business Ethics, 60(3), 221-232.

Williamson, D., Lynch-Wood, G. et RAMSEY, J. (2006). Drivers of environmental behaviors in manufacturing SMEs and the implications for CSR. Journal of Business Ethics, 3(67), 317-330.

Williamson, O.E. (1993). Opportunism and its critics. Managerial and Decision Economics, 14(2), 97-107.

YAMI, S. (2003). Petite entreprise et stratégie collective de filières. Revue française de gestion, 3(144), 165-179.

Yami, S. (2006). Fondements et perspectives des stratégies collectives. Revue française de gestion, 8(167), 91-104.

YIN, R.K. (1989). Case Study research, design and methods. Londres, Sage Publications. 